\title{
Ferromagnetic fraction and exchange anisotropy in SUS 316LN austenitic stainless steel due to strain-induced deformation
}

\author{
J. Manjanna, ${ }^{\text {a) }}$ Y. Kamada, S. Kobayashi, S. Takahashi, and H. Kikuchi \\ NDE and Science Research Center, Faculty of Engineering, Iwate University, Morioka 020-8551, Japan
}

(Presented on 6 November 2007; received 12 September 2007; accepted 14 October 2007; published online 24 January 2008)

\begin{abstract}
The exchange bias behavior was observed between strain-induced ferromagnetic (FM) $\alpha^{\prime}$ martensite and antiferromagentic (AFM) austenitic phase in SUS 316LN stainless steel at $<30 \mathrm{~K}$. Based on the magnetic hysteresis loops, the exchange contact was evident when a small amount of FM was formed, typically about $<1.0 \mathrm{vol} \%$. The exchange bias field $\left(H_{\mathrm{ex}}\right)$ was reported here as a function of FM volume fraction at different temperatures. The decreasing $H_{\mathrm{ex}}$ with FM is attributed to the increasing size of FM crystallites in the AFM matrix. () 2008 American Institute of Physics. [DOI: $10.1063 / 1.2830831]$
\end{abstract}

\section{INTRODUCTION}

SUS $316 \mathrm{LN}$ is a molybdenum bearing nitrogen-alloyed low-carbon austenitic steel. It is paramagnetic at room temperature and antiferromagnetic (AFM) below its blocking temperature $\left(T_{b}\right)$. Type $316 \mathrm{LN}$ alloy is used in widespread industrial and technological applications ranging from nuclear reactors to cryogenic equipments because of its superior mechanical and chemical properties. It is a candidate material for fusion reactors wherein the material must remain nonmagnetic to avoid interactions with the magnetic field produced by the superconducting magnets. ${ }^{1,2}$ Hence, it is important to investigate the magnetic behavior of this material especially in the cryogenic temperatures.

It is well known that strain-induced deformation can transform the austenitic structure (fcc, $\gamma$ ) to ferromagnetic (FM) $\alpha^{\prime}$ martensite (bct) in stainless steels, which depends on the chemical composition of the alloy, temperature, strain rate, etc. In type 316LN steel, a relatively stable alloy among 300 grade steels, such a $\gamma \rightarrow \alpha^{\prime}$ transformation was considered to be a hindered process when deformed at room temperature, unlike the low-temperature deformations. ${ }^{1,2}$

Exchange bias refers to the effect where the magnetization of a FM layer is pinned into a well-defined reference direction by an AFM such that its hysteresis loop exhibits a horizontal shift when field cooled from above $T_{b}$. Conventionally, exchange interaction was observed due to contact interface between the FM and AFM layer systems such as thin films, fine particles, and nanoparticles of FM core covered with AFM shell. ${ }^{3,4}$ However, such an exchange interaction is not commonly seen in the crystalline alloys which was first reported on cold-worked-type 347 stainless steel by Meiklejohn. ${ }^{4}$

Although few studies have mentioned such exchange interaction between the strain-induced FM and austenitic phase in stainless steels, ${ }^{5,6}$ there is no detailed information on the extent of such exchange contact as a function of $\alpha^{\prime}$ martensite volume fraction. In summary, the possible AFM/FM in-

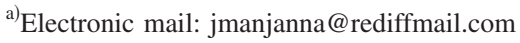

teractions in austenitic steels have received less attention. In this study, we have investigated this aspect using SUS 316LN alloy which was compressively deformed at room temperature (RT) and also at liquid- $\mathrm{N}_{2}$ temperature.

\section{EXPERIMENTAL PROCEDURE}

The chemical composition of the SUS 316LN alloy is shown in Table I. The samples machined to $3 \times 3 \times 2 \mathrm{~mm}^{3}$ were solution annealed at $1050{ }^{\circ} \mathrm{C}$ for $80 \mathrm{~min}$, water quenched and thereafter electropolished and cleaned. Then, compressively deformed at RT at a strain rate of $0.5 \mathrm{~mm} / \mathrm{min}$ and obtained different amounts of FM $(<0.2 \mathrm{vol} \%)$ corresponding to different true strains $\left(\varepsilon_{t}\right)$. To achieve higher amount of FM, a few samples were compressed at $77 \mathrm{~K}\left(\varepsilon_{t}=30 \%-50 \%\right)$ wherein about $1-5 \mathrm{vol} \%$ FM was obtained. Magnetization was measured between 2 and $300 \mathrm{~K}$ using a superconducting quantum interference device magnetometer. The field applied $(H)$ was perpendicular to the axis of compressive deformation.

During the hysteresis $(M-H)$ loop measurement, the applied field was cycled from 30 to $-30 \mathrm{kOe}$. The exchange contact was observed in the field-cooled (FC) condition wherein the sample was cooled from $300 \mathrm{~K}$ to different low temperatures at $H_{\mathrm{FC}}=30 \mathrm{kOe}$. In zero-field-cooled (ZFC) condition, the sample was cooled from 300 to $2 \mathrm{~K}$ at $H=0$ and then rose to different temperatures.

Exchange bias field $\left(H_{\mathrm{ex}}\right)=\left(H_{c_{L}}-H_{c_{R}}\right) / 2$ where $H_{c_{L}}$ and $H_{c_{R}}$ are the left and the right intercepts of the $M-H$ loop with the field axis. In this study, $H_{\mathrm{ex}}$ was obtained after subtract-

TABLE I. Chemical composition of SUS 316LN (wt \%).

\begin{tabular}{cccccc}
\hline \hline $\mathrm{C}$ & $\mathrm{Si}$ & $\mathrm{Mn}$ & $\mathrm{P}$ & $\mathrm{S}$ & $\mathrm{Ni}$ \\
\hline 0.015 & 0.58 & 1.39 & 0.022 & $<0.0005$ & 12.71 \\
$\mathrm{Cr}$ & $\mathrm{Mo}$ & $\mathrm{N}$ & $\mathrm{Co}$ & $\mathrm{Cu}$ & $\mathrm{Fe}$ \\
\hline 17.29 & 2.52 & 0.112 & 0.04 & 0.08 & $\mathrm{Bal}$. \\
\hline \hline
\end{tabular}




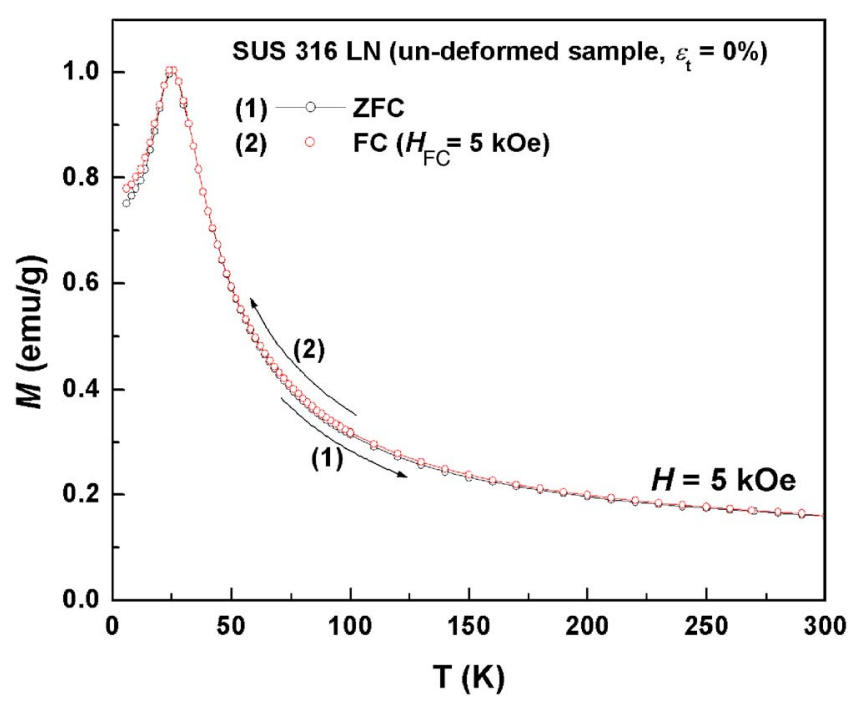

FIG. 1. (Color online) Magnetization of the undeformed sample indicating the paramagnetic and antiferromagnetic behaviors as a function of temperature.

ing the magnetization of austenite matrix (equivalent to the magnetization of the undeformed sample at the same condition that used for deformed samples).

\section{RESULTS AND DISCUSSION}

The blocking temperature $\left(T_{b}\right)$ of SUS $316 \mathrm{LN}$ was determined to be $\approx 30 \mathrm{~K}$ as indicated from the magnetization plots of undeformed sample as a function of temperature under ZFC and FC conditions (Fig. 1). Thus, the alloy is paramagnetic above $30 \mathrm{~K}$ and below which it is AFM.

As shown in Fig. 2, the amount of FM $\alpha^{\prime}$ martensite increased sharply after about $30 \%$ true strain as determined from the saturation magnetization $\left(M_{s}\right)$ measured at RT. In these steels, austenite to $\alpha^{\prime}$ martensite transformation is believed to follow ${ }^{7,8}$ as $\gamma($ fcc $) \rightarrow$ stacking faults $\rightarrow \varepsilon$ (hcp) $\rightarrow \alpha^{\prime}$ (bct). Further, it is known that $\alpha^{\prime}$-martensite grains ap-

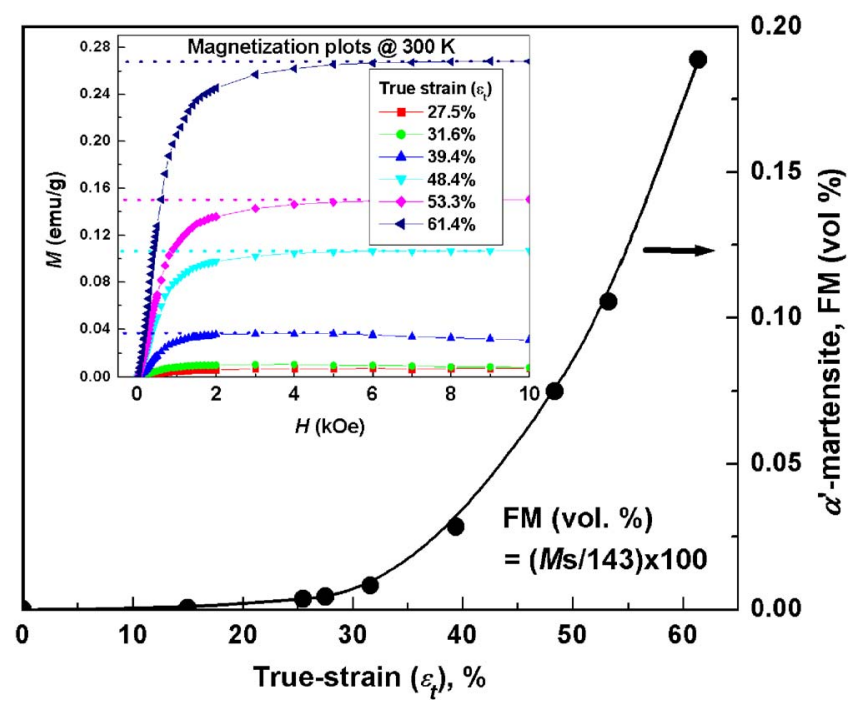

FIG. 2. (Color online) Volume fraction of strain-induced $\alpha^{\prime}$ martensite (FM) due to compressive deformation at RT. The inset shows the saturation magnetization plots measured at RT and after subtracting the magnetization of the austenitic matrix.

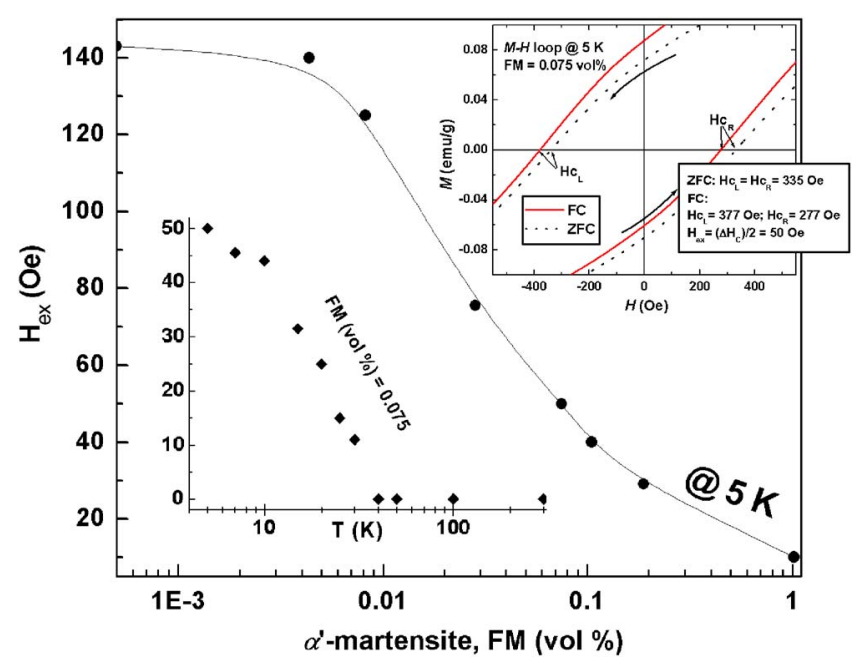

FIG. 3. (Color online) $H_{\mathrm{ex}}$ as a function of FM volume fraction at $5 \mathrm{~K}$. The top inset is the enlarged view of the $M-H$ loops, and the bottom inset is the $H_{\mathrm{ex}}$ vs $T$ for the sample deformed to $\varepsilon_{t}=48.4 \%$.

pear inside the austenite grains mainly at the intersection of shear bands, making the movement of dislocations more difficult.

The FM is presumed to be distributed homogeneously in the austenite matrix at this small amounts $(<0.2$ vol \%). When hysteresis loops were measured for such coexisting system, the symmetrical loop under ZFC became asymmetrical if field cooled through $T_{b}$. In a typical case as shown in the inset (top) of Fig. 3, it is apparent that the loop is shifted in the horizontally negative direction. The origin of this shift along the field axis is ascribed to the exchange contact at the boundary or interface of $\alpha^{\prime}$ martensite in austenitic matrix, ${ }^{4-6}$ i.e., the microscopic torque is exerted by the AFM spins to the spins in the FM while reversing the magnetization of FM.

Since $H_{\mathrm{ex}}$ depends on the cooling field $\left(H_{\mathrm{FC}}\right)$, in this study, it was determined to be about $30 \mathrm{kOe}$ as the $H_{\mathrm{FC}}$ and remanence reached a steady state.

Figure 3 shows $H_{\text {ex }}$ as a function of FM volume fraction at $5 \mathrm{~K}$, as a typical case. It is found that $H_{\mathrm{ex}}$ decrease from about 143 to $10 \mathrm{Oe}$ as the FM was increased to about 1 vol \% from a negligibly small amount. A similar behavior was observed at all the temperatures from 2 to $30 \mathrm{~K}$, and thereafter the $M-H$ loops were symmetrical and hence $H_{\mathrm{ex}}$ was zero. For comparison of the $H_{\mathrm{ex}}$ as a function of FM volume fraction, in Fig. $3, H_{\mathrm{ex}}$ of the samples deformed at RT $(\mathrm{FM}<0.2$ vol \%) are extrapolated with the sample deformed at $77 \mathrm{~K}(\mathrm{FM} \approx 1.0 \mathrm{vol} \%)$ even though the microstructure of FM was expected to be different. ${ }^{1}$

The bottom inset in Fig. 3 shows $H_{\mathrm{ex}}$ as a function of temperature for the sample deformed to $48.4 \%$ true strain. It is found that $H_{\mathrm{ex}}$ decreases from 50 to $10 \mathrm{Oe}$ as the temperature approaches $30 \mathrm{~K}$ (the so-called $T_{b}$ ) from $5 \mathrm{~K}$. This behavior is in corroboration with Fig. 1, and verifies that the exchange bias phenomenon in SUS 316LN system exists for $T<T_{b}$. This decreasing trend of $H_{\text {ex }}$ with increasing temperature remained same for all other samples deformed to different true strains $(\mathrm{FM}<1$ vol \%). The temperature dependent 
feature of the exchange coupling can be qualitatively explained based on the thermal fluctuation of the magnetic moments.

It is clear from Fig. 3 that the exchange contact was established only when small volume fraction of FM was formed in austenitic structure, typically about $<1.0 \mathrm{vol} \%$, and beyond which $H_{\mathrm{ex}}$ was found to vanish.

The variation of $H_{\mathrm{ex}}$ with increasing FM amount may be explained based on the increasing size of $\alpha^{\prime}$ martensite in AFM austenitic matrix. The $\alpha^{\prime}$-martensite crystallites, once nucleated, are distributed wide apart in the austenite matrix establishing intimate contact with AFM phase. Thus, in the initial stage, the ratio of interface area between FM sites and AFM matrix is higher and hence the higher exchange contact. Due to small deformation $\left(\varepsilon_{t}<30 \%\right)$, it seems that the number density of isolate $\alpha^{\prime}$ martensite increase while the size remain more or less same. On further deformation $\left(\varepsilon_{t}\right.$ $>30 \%$ ), the size of FM sites was expected to increase. Thus, the relative interface area decreased, consequently, $H_{\mathrm{ex}}$ decreased with increasing FM. Nevertheless, the detailed mechanism regarding how the microstructure can affect the $H_{\mathrm{ex}}$ is not clearly understood yet. For layered structures, competing models for the origin of $H_{\mathrm{ex}}$ have been proposed: "spin flop" model assumes a $90^{\circ}$ angle between the magnetization of FM and AFM, ${ }^{9}$ and coupling by uncompensated moments at steps or grain boundaries. ${ }^{10}$ Further, as the exchange interaction seems to derive from the interface between FM and AFM components, it was regarded that interface disorder such as roughness, crystallinity, grain size, and interface impurity, could play an important role on the extent of exchange coupling. ${ }^{11}$ The martensite cluster size at this small amounts $(<1 \mathrm{vol} \%)$ is presumed to be of the order of nanometer scale; however, any experimental evidence on the microstructure of $\alpha^{\prime}$ martensite could not be obtained (using transmission electron micrography and X-ray diffraction) at this small volume fraction, ${ }^{12}$ and also due to high dislocation density caused by deformation.

In summary, this study has clearly shown that the exchange anisotropy can be observed only when small amount of $\alpha^{\prime}$ martensite (less than about $1.0 \mathrm{vol} \%$ ) was formed in the austenitic steel, SUS 316LN. As shown in Fig. 3, $H_{\mathrm{ex}}$ decreased with increasing amount of FM, and increased as the temperature was lowered below $T_{b}$. The dependence of $H_{\mathrm{ex}}$ with the volume fraction of FM is attributed to the increasing size of FM sites in the surrounding AFM austenite matrix.

${ }^{1}$ J.-B. Vogt, J. Foct, C. Regnard, G. Robert, and J. Dhers, Metall. Trans. A 22A, 2385 (1991).

${ }^{2}$ M. Botshekan, S. Degallaix, Y. Desplanques, and J. Polak, Fatigue Fract. Eng. Mater. Struct. 21, 651 (1998).

${ }^{3}$ J. Nogues, J. Sort, V. Langlais, S. Doppiu, B. Dieny, J. S. Munoz, S. Surinach, M. D. Baro, S. Stoyanov, and Y. Zhang, Int. J. Nanotechnol. 2, 23 (2005).

${ }^{4}$ W. H. Meiklejohn, J. Appl. Phys. 32, S274 (1961).

${ }^{5}$ J. Crangle, A. Fogarty, and M. J. Taylor, J. Magn. Magn. Mater. 111, 255 (1992).

${ }^{6}$ J. Ding, H. Huang, P. G. McCormic, and R. Street, J. Magn. Magn. Mater. 139, 109 (1995).

${ }^{7}$ W. Hübner, Tribol. Int. 34, 231 (2001).

${ }^{8}$ R. P. Reed, Acta Metall. 10, 865 (1962).

${ }^{9}$ N. C. Koon, Phys. Rev. Lett. 78, 4865 (1997).

${ }^{10}$ K. Takano, R. H. Kodama, A. E. Berkowitz, W. Cao, and G. Thomas, Phys. Rev. Lett. 79, 1130 (1997).

${ }^{11}$ J. Nogues and I. K. Schuller, J. Magn. Magn. Mater. 192, 203 (1999).

${ }^{12}$ K. Mumtaz, S. Takahashi, J. Echigoya, L. F. Zhang, Y. Kamada, and M. Sato, J. Mater. Sci. 38, 3037 (2003). 